\title{
Neumoperitoneo hipertensivo y síndrome compartimental abdominal causado por perforación de un vólvulo gástrico
}

\author{
Dr. Salomón T. Domínguez-Pérez, ${ }^{*}$ Dr. Carlos Baeza-Herrera, ${ }^{* *}$ Dr. Ricardo Cortés-García, ${ }^{* * *}$ Dra. Alin \\ Villalobos-Castillejos, ${ }^{* * *}$ CD. J. Marcos R. Aguilar-Venegas ${ }^{* * * *}$
}

\section{RESUMEN}

Introducción: El neumoperitoneo hipertensivo es extraordinariamente raro. Los informes señalan que ocurre en neonatos o lactantes y que es consecuencia de una perforación intestinal, de enterocolitis necrosante o de una invaginación intestinal. El desarrollo de un síndrome compartimental abdominal ulterior es un evento inusual.

Presentación del caso: Niña de diez años de edad que ingresó por dolor abdominal, de evolución tórpida, con gran distensión gástrica, que desencadenó alteraciones hemodinámicas graves. Se decidió operarla, durante la laparotomía se encontró una perforación gástrica causada por un vólvulo mesenterioaxial. Se realizó el cierre del estómago y se hizo una gastrostomía. Al año de la cirugía la paciente se encuentra estable.

Palabras clave: Neumoperitoneo hipertensivo, síndrome compartimental abdominal, vólvulo gástrico, perforación gástrica.

\section{ABSTRACT}

Introduction: Hypertensive pneumoperitoneum is an extraordinary event. It has been reported in neonates and toddlers, associated with intestinal perforation due to necrotizing enterocolitis or to intussusception. However, secondary abdominal compartment syndrome is quite unusual.

Case report: A 10 year-old female presented with abdominal pain. She developed abdominal distention and hemodynamic instability. A laparotomy was performed and a gastric perforation secondary to a gastric volvulus was found. The gastric perforation was repaired and a gastrostomy was performed. At one year of the surgery, the patient is recovering well.

Key words: Hypertensive pneumoperitoneum, abdominal compartment syndrome, gastric volvulus, gastric perforation.

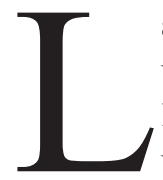

a presencia de aire libre subdiafragmático es una entidad que se diagnostica clínica y radiológicamente. Es causada por la perforación de una víscera hueca. Su tratamiento es quirúrgico

* Cirujano Pediatra Adscrito. Hospital Pediátrico Moctezuma

** Jefe Cirugía Pediátrica. Hospital Pediátrico Moctezuma.

*** Residentes de Cirugía Pediátrica. Hospital Pediátrico Moctezuma.

**** Departamento de Atención a la Salud. Universidad Autónoma Metropolitana-Xochimilco.

Correspondencia Dr. Salomón T. Domínguez Pérez. Hospital Pediátrico Moctezuma. Oriente 158 No. 189. Col. Moctezuma 2a. Sección. Venustiano Carranza 15530. México D.F. 557140 57, Faz 557117 37. Correo electrónico: salomondominguez@hotmial.com Recibido: octubre, 2009. Aceptado: diciembre, 2009.

Este artículo debe citarse como: Domínguez-Pérez ST, BaezaHerrera C, Cortés-García R y col. Neumoperitoneo hipertensivo y síndrome compartimental abdominal causado por perforación de un vólvulo gástrico. Acta Pediatr Mex 2010;31(2):60-63.

www.nietoeditores.com.mx
${ }^{1}$. No existe una clasificación para graduar la gravedad del problema. El neumoperitoneo a tensión se ha descrito en casos de invaginación intestinal, al realizar neumoenemas como tratamiento de la enfermedad ${ }^{2,3}$. Las consecuencias de la hipertensión intraabdominal se empezaron a documentar a finales del siglo XIX, en casos de muerte por disfunción respiratoria ${ }^{4}$. Las alteraciones cardiovasculares y renales secundarias, fueron descritas desde $1911^{4}$. En pediatría, se ha descrito el síndrome compartimental abdominal principalmente en neonatos, a consecuencia de la corrección de defectos de la pared abdominal como el onfalocele y la gastrosquisis; con menos frecuencia, como consecuencia de eventos traumáticos ${ }^{5-7}$. El tratamiento médico y principalmente quirúrgico de este síndrome, es un reto, particularmente en pacientes pediátricos ${ }^{6-8}$.

\section{CASO CLÍNICO}

Niña de diez años de edad, sin antecedentes de importancia. Proviene de un medio socioeconómico pobre. Seis 
meses antes de su ingreso, estuvo internada en nuestra unidad por dolor abdominal intenso, vómito y fiebre. Ingresó al servicio de terapia intensiva con diagnóstico de probable pancreatitis. Una pancreatografía dinámica, descartó dicha patología, pero permitió observar una elevación del hemidiafragma izquierdo. Una endoscopia no halló anormalidades. Ingresó nuevamente por dolor abdominal de $24 \mathrm{~h}$ de evolución, en el epigastrio, tipo cólico, sin irradiación; aumentó en intensidad gradualmente hasta volverse intolerable. Se acompañó de náusea y vómito en diez ocasiones, de contenido gástrico escaso. El tratamiento con butilhioscina no alivió el dolor.

Examen Físico. La niña estaba consciente, regularmente hidratada; facies de dolor y en posición incómoda por el mismo. Peso $40 \mathrm{~kg}$; talla $142 \mathrm{~cm}$. FC: $85^{\circ}$ FR: 30“, Temp: $37^{\circ} \mathrm{C}$; TA 100/60 mmHg. Perímetro abdominal, $67 \mathrm{~cm}$. Normocéfala, cardiorrespiratorio sin compromiso, abdomen distendido. Dolor difuso epigástrico con irradiación a la región lumbar; timpánico a la percusión; no se palparon organomegalias. Motilidad intestinal muy disminuida. Genitales normales. Extremidades sin alteraciones; llenado capilar de dos segundos. El aumento del dolor y la náusea, hicieron colocar una sonda nasogástrica, por la cual se obtuvo escaso gasto gástrico.

Laboratorio. La biometría mostró 9,800 leuc/ $\mathrm{mm}^{3}$; $\mathrm{Hb}, 14.9 \mathrm{mg} / \mathrm{dL}$; Hto, 44.7\%; eritrocitos, 4,550,000/. $\mathrm{mm}^{3}$;

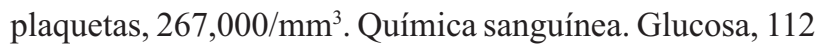
mg; creatinina, 0.57; urea, 12. Electrólitos séricos. Na, 133 meq; K, 3.4 meq; cloro, 98 meq; Ca, 8.1 meq. Función hepática. AST: 28, ALT: 36, BT: 0.69. Amilasa 62, TP: $14.4 \mathrm{seg}$, TTP: 33.9 seg INR: 1.19.

Seis horas después de su ingreso aumentó súbitamente el dolor abdominal, con irradiación a ambos hombros y diaforesis profusa. La paciente estaba soporosa, pálida, con cianosis distal, disneica. Tenía distensión abdominal con incremento del perímetro abdominal de $10 \mathrm{~cm}$; no era audible la peristalsis, había timpanismo en ambos flancos; las extremidades inferiores estaban marmóreas, frías, no se palpaban los pulsos dístales. FC: 110', FR: 49“. TA: 90/ $50 \mathrm{mmHg}$ en miembro superior; no palpable en el inferior. Hemograma de control. Hb 9.4 mg/dL; Hto 28.2\%. Leucocitos $13,400 / \mathrm{mm}^{3}$. Gasometría arterial. Acidosis mixta no compensada. Las radiografías de abdomen mostraban aire libre subdiafragmático, con desplazamiento del hígado a la línea media (Figura 1). Antes de la laparotomía exploradora, se introdujo un punzocath 17 Fr. en flanco izquierdo (Figura

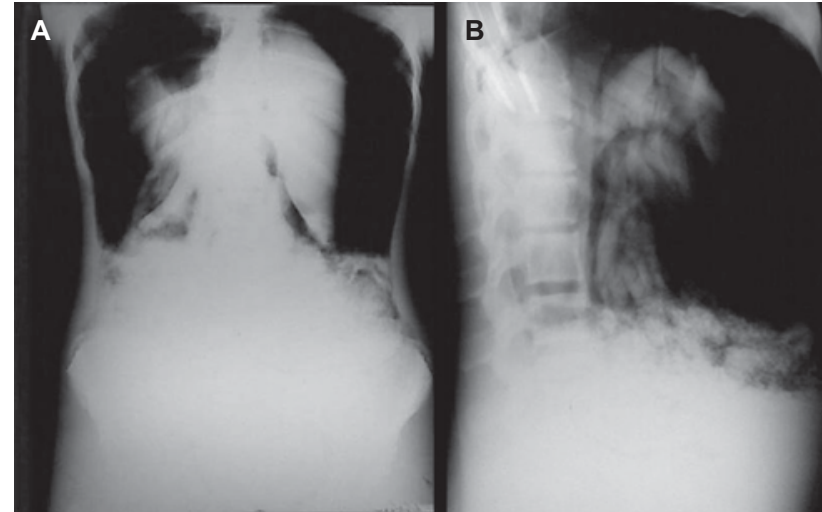

Figura 1A. A la izquierda, radiografía AP de abdomen que muestra un neumoperitoneo masivo que desplaza la glándula hepática hacia abajo y hacia la línea media. B. A la derecha, radiografía lateral de abdomen que muestra las asas de intestino rechazadas.

2), que permitió evacuar el aire intraperitoneal y estabilizar hemodinámicamente a la paciente. Durante la cirugía se halló una perforación en la cara anterior de la curvatura mayor del estómago, (Figura 3), causada por un vólvulo mesenterioaxial que se clasificó como vólvulo gástrico idiomático; había escaso líquido libre en "pozos de café".

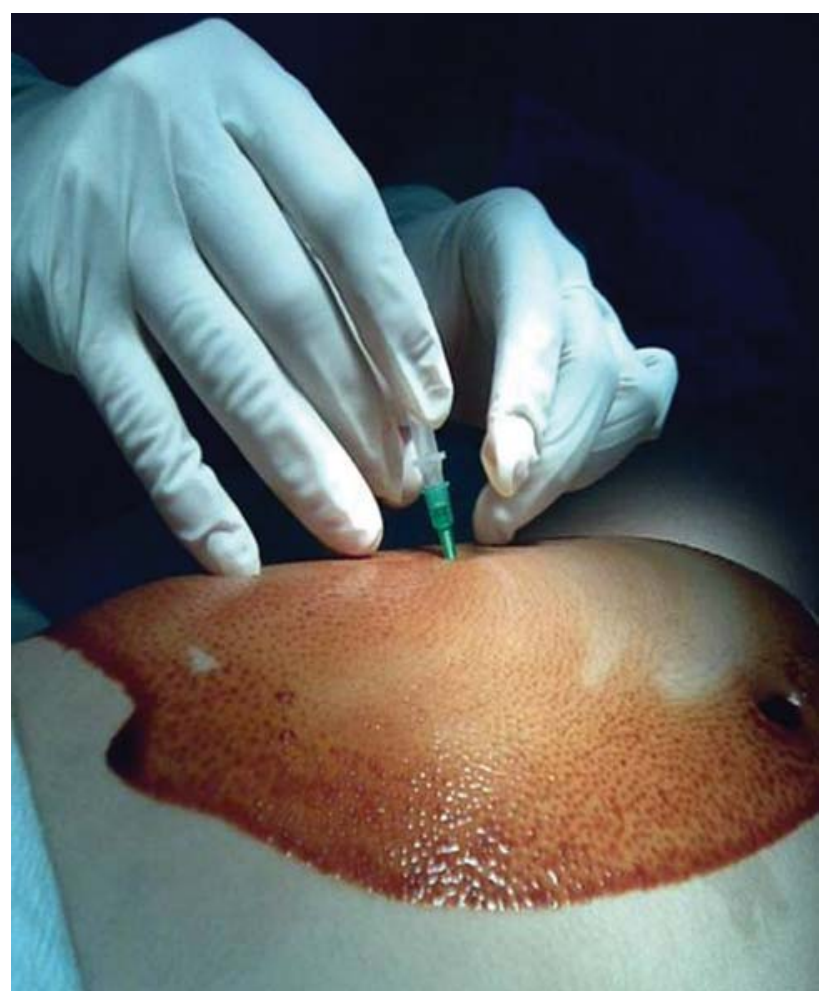

Figura 2. Punción intraperitoneal evacuadora con punzocath No. 17. 


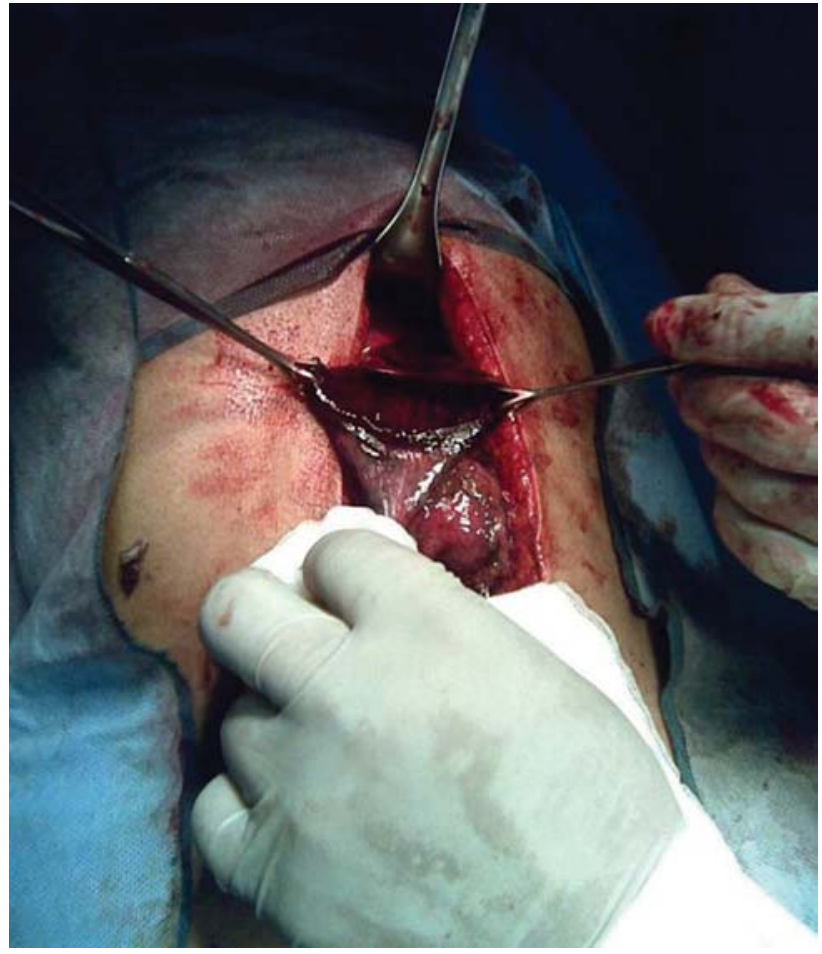

Figura 3. Obsérvese la magnitud de la perforación gástrica.

Se realizó cierre primario del estómago (Figura 4) y enseguida, una gastrostomía. No se encontraron defectos asociados. En el postoperatorio la paciente recibió doble esquema antimicrobiano por cinco días, apoyo ventilatorio y aminas por 12 horas.

Los estudios preoperatorios para identificar la causa de la perforación gástrica fueron negativos: Las radiografías de control, no mostraron elevación del hemidiafragma izquierdo. La evolución postoperatoria fue satisfactoria; la paciente empezó a comer dieta al tercer día posoperatorio y egresó al quinto día. Durante el control por la consulta externa a un año de la cirugía, la paciente se encontró estable.

\section{DISCUSIÓN}

La presencia de neumoperitoneo a tensión con desarrollo de síndrome compartimental abdominal secundario es un evento raro y poco referido en la literatura. En 1980 se acuñó el término para este síndrome ${ }^{4}$ y se empezaron a describir las alteraciones fisiológicas debidas al aumento de presión intraabdominal (PIA). No existe una cifra de la PIA que señale el momento preciso en que inicia este sín-

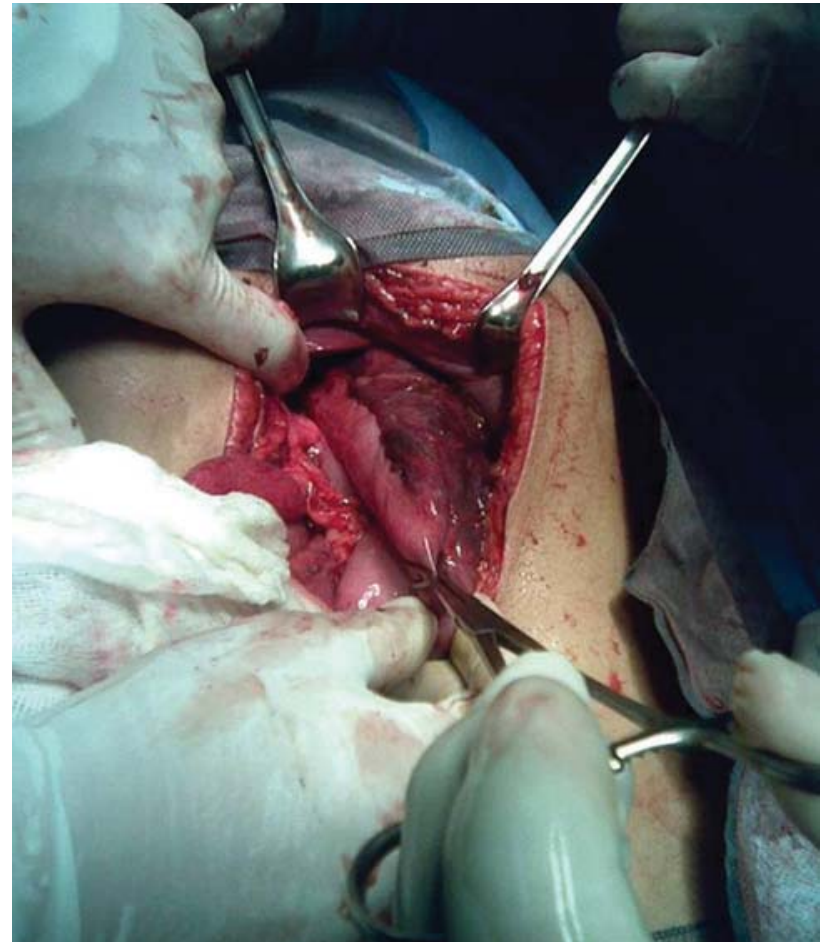

Figura 4. Gastroplastia de la curvatura mayor.

drome ${ }^{8-11}$. Sin embargo, se considera que hay un aumento importante de la presión abdominal cuando se registran cifras $\geq 12 \mathrm{mmHg}$ en tres medidas espaciadas en un lapso de 4 a 6 h y se desarrolla un síndrome compartimental abdominal cuando la PIA es de $\geq 20 \mathrm{mmHg}$ en tres medidas durante un lapso de 1 a 6 h, y ocurre una disfunción orgánica causada por la reducción del aporte sanguíneo de los órganos intraabdominales, aparecen compromiso ventilatorio restrictivo y colapso cardiovascular ${ }^{6,8-10}$. Por otra parte, el desarrollo súbito del síndrome puede sospecharse cuando además de la hipertensión intraabdominal se acompaña de gran distensión abdominal, imposibilidad de palpar los pulsos femorales; cianosis y enfriamiento de las extremidades inferiores; oliguria progresiva e hipoxia por las alteraciones respiratorias secundarias. Todos estos son elementos que hacen necesaria una descompresión abdominal ${ }^{11-13}$.

No se conoce el mecanismo mediante el cual el aire libre subdiafragmático en gran cantidad provoca alteraciones hemodinámicas que ponen en riesgo la vida de los pacientes. Algunos autores piensan que es un mecanismo de válvulas, que se forma en el sitio donde existe la fuga aérea hacia la cavidad abdominal, lo que produce una 
diferencia de presión entre la cavidad peritoneal y el resto del tubo digestivo, debido al flujo aéreo directo hacia el interior del peritoneo, sin permitir su salida $3,14,15$. Esto provoca compresión diafragmática y secundariamente, restricción respiratoria con dificultad y estasis del retorno circulatorio y falla cardiaca, desplazamiento del hígado que también contribuye a la disminución del retorno circulatorio, con repercusión sobre las extremidades ${ }^{3,7,9-13}$. La evacuación de la cantidad masiva de aire intraperitoneal con un punzocath, es una medida heróica, y eficaz ${ }^{12,13}$. En nuestra paciente se logró una estabilidad hemodinámica transitoria, que permitió realizar de inmediato la intervención quirúrgica. La causa de esta patología fue un vólvulo gástrico, infrecuente en pacientes pediátricos ${ }^{16,17}$.

\section{BIBLIOGRAFÍA}

1. Krauz M, Manny J. Pneumoperitoneum associated with pneumotorax. A surgical dilemma in the post-traumatic patient. $J$ Trauma. 1977;17:238-40.

2. Addison NV, Brougthon AC. Tension pneumoperitoneum: a report of 4 cases. Br J Surg. 1976;63:877-80.

3. Baeza HC, Herrera PV, García CH J, González OA. Neumoperitoneo hipertensivo. Una causa inesperada de muerte. Rev Ibero Cuid Int. 1997;6:98-101.

4. Burch JM, Moore EE, Moore FE. The abdominal compartment syndrome. Surg Clin North Am. 1996;76:833-42.

5. Morrell BJ, Vinden C, Singh RN, et al. Secondary abdominal compartment syndrome in a case of pediatric trauma shock resuscitation. Ped Crit Care Med. 2007;8:67-70.
6. Carlotti AP, Carvalho WB. Abdominal compartment syndrome: A review. Ped Crit Care Med. 2009;10:115-20.

7. Kawar B, Siplovich L. Abdominal compartment syndrome in children: The dilemma of treatment. Eur J Ped Surg. 2003;13:330-33.

8. Tomicic FV, Cruces RP, Donoso FA. Síndrome compartimental del abdomen en el paciente crítico. Rev Chil Pediatr. 2006;77:557-67.

9. Malbrain CL, Chiumello D, Pelosi P, et al. Prevalence of intraabdominal hypertension in critically ill patients: a multicenter epidemiological study. Intensive Care Med. 2004;30:822-29.

10. Castellanos G, Piñeiro A, Fernández J. La hipertensión intraabdominal y el síndrome compartimental abdominal: ¿Qué debe saber y cómo debe tratarlo el cirujano? Cir Esp. 2007;81:4-11.

11. Robert BR, Blake B, Bruggeman GE. Tension pneumoperitoneum: a case of ventilatory obstruction. Anesthesiology. 1981;55:326-7.

12. Kim HB, Lillehei CW. Life threating tension pneumoperitoneum from intestinal perforation during air reduction of intussusception. Paediatric Anest. 2002;12:798-800.

13. Fernándes ML, Pires KC, Chimelli PH. Síndrome de compartimiento abdominal, durante pinzamiento por vía endoscópica de perforación intestinal secundaria a la colonoscopía. Rev Bra Anestesiol. 2009;59:614-7.

14. Ramírez CJ, Rodríguez BI, Rodríguez BR. Neumoperitoneo neonatal secundario a la ventilación mecánica intermitente. Pediatr. 1993;60:53-7.

15. Ilgen EB, Symchych PS. Pneumoperitoneum without ruptured viscus in the neonate: A case report and review of the literature. J Ped Surg. 1997;12:537-40.

16. Faray S, Friallo V, Nash S. Gastric perforation in a case of gastric volvulus. Am J Gastroent. 1996;91:1863-4.

17. Mayo A, Erez I, Lazar $L$ et al. Volvulus of the stomach in children: the spectrum of the disease. Ped Emerg Care. 2001;17:344-48-

\section{FE DE ERRATA}

En el volumen 31, número 1, año 2010, el artículo "Tratamiento de los quistes de colédoco en la edad pediátrica: Una revisión de 24 años" tiene dos errores:

En el nombre del primer autor

DICE: Dra. José David Palmer Becerra

DEBE DECIR: Dr. José David Palmer Becerra

En el primer párrafo de la Introducción

DICE: Los quistes se dividen en seis tipos

DEBE DECIR: Los quistes se dividen en cinco grupos.

Ofrecemos una disculpa al Dr. Palmer-Becerra por los errores involuntarios en su artículo. Unidad de Publicaciones Médicas 